\title{
Urinary Bisphenol A in PCOS women and its relation to steroid hormones
}

\section{Lazúrová Z. 1, Hubková B. ${ }^{2}$, Mareková M. ${ }^{2}$, Figurová J. ${ }^{1}$, Lazúrová I. ${ }^{1}$}

11 st Department of Internal medicine, L. Pasteur University Hospital and Medical faculty of P.J. Šafárik University, Košice

2 Department of Clinical Biochemistry, L. Pasteur University Hospital and Medical faculty of P.J. Šafárik University, Košice

\section{OBJECTIVES}

Bisphenol A (BPA) is an environmental estrogen found in plastic material such as bottles, food package, dental metarial, etc. It is known to have negative effects on human health and its exposure is associated with some endocrine disorders including polycystic ovary syndrome (PCOS). Some studies demonstrated significantly higher serum levels of BPA in PCOS women and its association with serum androgens, whereas some did not confirm these data in PCOS. The association between BPA and steroid hormones is still controversial.

\section{AIM}

\section{METHODS}

- Study sample:

- 69 women with PCOS according to AES criteria

- 38 healthy age-matched regularly menstruating controls

- Methods:

fasting glycemia, insulin, lipid profile and hormonal profile (testosterone, f-testosterone, androstendione, dihydrotestosterone (DHT), DHEAS, estrone) were evaluated

urinary BPA concentrations were measured in spot urine samples using high pressure liquid chromatography

To compare urinary BPA concentrations between PCOS and healthy non PCOS women and to assess relationship between BPA and ovarian and adrenal steroid hormones in PCOS group.

Tab. 1. Measured data in PCOS women and controls. Tab. 2. Measured parameters in group of PCOS women, and subgroups with low

\begin{tabular}{cccc}
\hline Parameter & $\begin{array}{c}\text { PCoS } \\
(\mathrm{n}=69)\end{array}$ & $\begin{array}{c}\text { Controls } \\
(\mathrm{n}=38)\end{array}$ & Significance \\
\hline Age & $29.9 \pm 5.2$ & $28 \pm 6.4$ & NS \\
\hline BMI (kg/m $\left./ \mathrm{m}^{2}\right)$ & $27.7 \pm 7$ & $20.7 \pm 2.1$ & 0.01 \\
\hline BPA (ug/g creat ) & $6.8 \pm 9.8$ & $5.7 \pm 9.6$ & NS
\end{tabular}
and higher levels of BPA in urine (BPA neg, BPA posit).

Figure 1. Correlation between DHEAS and BPA in

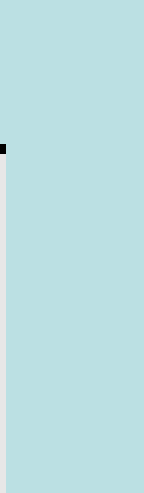

\begin{tabular}{|c|c|c|c|c|c|c|c|c|c|}
\hline Parameter & $\begin{array}{c}\text { PCOS } \\
(n=69)\end{array}$ & $\begin{array}{c}\text { BPA } 0-0.5 \\
\text { ug/g creat } \\
(n=19)\end{array}$ & $\begin{array}{c}\text { BPA }>0.5 \\
\text { ug/g creat } \\
(n=50)\end{array}$ & $\mathbf{p}$ & Parameter & $\begin{array}{l}\text { PCOS } \\
(n=69)\end{array}$ & $\begin{array}{c}\text { BPA } 0-0.5 \\
\text { ug/g creat } \\
(n=19)\end{array}$ & $\begin{array}{c}\text { BPA }>0.5 \\
\text { ug/g creat } \\
(n=50)\end{array}$ & $\mathbf{p}$ \\
\hline BMI & $27.7 \pm 7$ & $27.7 \pm 7.6$ & $27.1 \pm 7$ & NS & FAI & $11.3 \pm 11.1$ & $12.5 \pm 10$ & $11 \pm 11$ & NS \\
\hline G & $5.1 \pm 1.6$ & $4.9 \pm 0.5$ & $5.2 \pm 1$ & NS & Androstendione & $5.0 \pm 1.5$ & $4.7 \pm 1.3$ & $5.2 \pm 1.6$ & NS \\
\hline
\end{tabular}
PCOS women
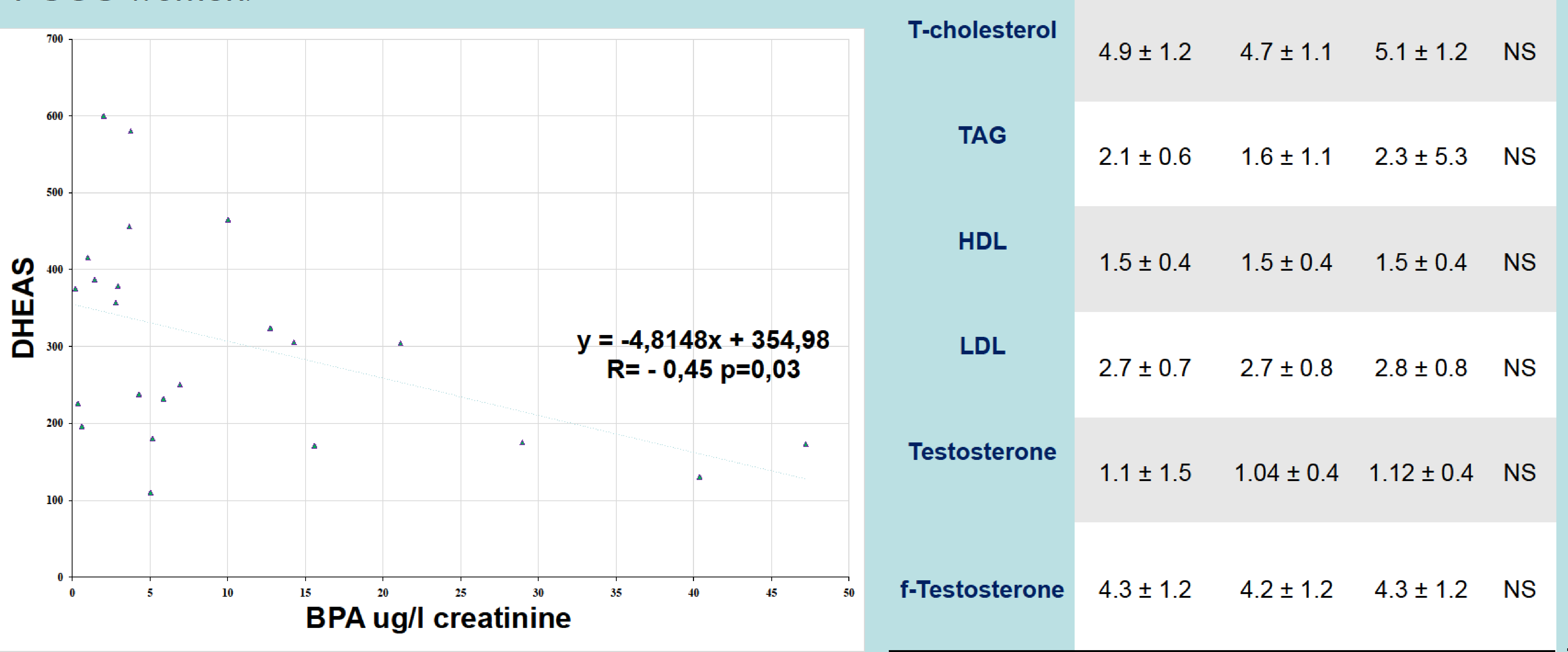

$254 \pm 194 \quad 333 \pm 195 \quad 311 \pm 136 \quad$ NS $114 \pm 129 \quad 153 \pm 172 \quad 96 \pm 100 \quad$ NS Estron

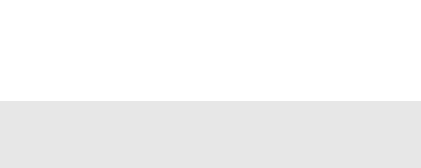

DHT
Androstan
Insulin

$682 \pm 449 \quad 767 \pm 565 \quad 642 \pm 383 \quad$ NS $6.1 \pm 4.8 \quad 7.4 \pm 6.4 \quad 5.6 \pm 3.8 \quad$ NS $4.4 \pm 9.7 \quad 9.9 \pm 5.8 \quad 16.1 \pm 10.4 \quad 0.06$ HOMA-IR $3.2 \pm 9.7 \quad 2.13 \pm 1.3 \quad 3.64 \pm 2.6 \quad 0.08$

\section{RESULTS}

Age, BMI and urinary BPA levels are shown in the Tab.1. As expected PCOS women had significantly higher BMI as compared with controls. Urinary BPA concentration was slightly, but not significantly higher in PCOS group compared to controls $(6.8 \pm 1.1 \mathrm{v} . \mathrm{s}$. $5.7 \pm 1.3$ ug/l creatinine) (Tab.1). When devided PCOS women according to the urinary levels of BPA into two groups, i.e. BPA positive (urinary BPA > $0.5 \mathrm{ug} / \mathrm{l}$ creatinine) and BPA negative (urinary BPA between 0-0.5 ug/l creatinine), there were no significant differences in measured hormones between two groups (Tab.2). Borderline statistical significance has been detected in serum insulin levels $(p=0.06)$ and HOMA-IR $(p=0.08)$ being slightly higher in BPA positive group. In the PCOS group no significant correlation has been detected between BPA and testosterone, free-testosterone, ASD, DHT and estradiol (all p>0.05), respectively. However, we found a significant negative correlation between BPA and DHEAS $(r=-0.59, p=0.0065)$ and a borderline negative correlation between BPA and serum DHT $(r=-0.23, p=0.07)$. Negative correlation between BPA and DHEAS is shown in Fig. 1. 